\title{
Spin-phonon interaction induces tunnel splitting in single-molecule magnets
}

\author{
Kilian Irländer ${ }^{1}$ and Jürgen Schnack ${ }^{1, *}$ \\ ${ }^{1}$ Fakultät für Physik, Universität Bielefeld, Postfach 100131, D-33501 Bielefeld, Germany
}

(Dated: March 25, 2022)

\begin{abstract}
Quantum tunneling of the magnetization is a major obstacle to the use of single-molecule magnets (SMMs) as basic constituents of next-generation storage devices. In this context, phonons are often only considered (perturbatively) as disturbances that promote the spin system to traverse the anisotropy barrier. Here, we demonstrate the ability of phonons to induce a tunnel splitting of the ground doublet which then reduces the required bistability due to Landau-Zener tunneling of the magnetization. Harmful are those phonons that modify the spin Hamiltonian so that its rotational symmetry about the field axis is destroyed. In our calculations we treat spins and phonons on the same footing by performing quantum calculations of a Hamiltonian where the single-anisotropy tensors are coupled to harmonic oscillators.
\end{abstract}

\section{INTRODUCTION}

Single-molecule magnets (SMMs) constitute magnetic materials in which the sufficiently separated molecules exhibit a magnetic hysteresis of purely molecular origin. Typically, such magnetic molecules are characterized by low-lying magnetic levels whose energies form a barrier against magnetization reversal. This situation is sketched in Fig. 1. Initialized in one of the two magnetic ground states, the system shows bistability and thus allows the storage of information in the same way a bit on a hard drive would do. Ever since the discovery of such behavior in $\mathrm{Mn}_{12}$-acetate [1-7], this promises future miniaturization of magnetic storage devices.

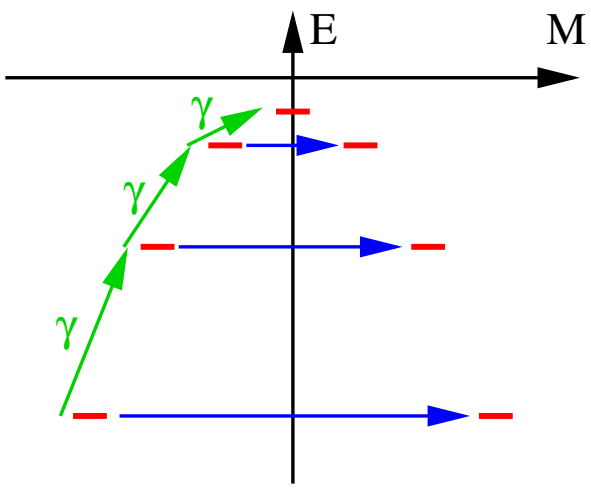

Figure 1. Sketch of the low-lying energy levels of an SMM vs. magnetic quantum number. Red bars denote energy eigenvalues; they form an anisotropy barrier. The tiny (invisible) energy differences between almost degenerate states for negative and positive magnetic quantum number, respectively, are called tunnel splitting. Blue arrows show magnetization tunneling pathways for states with negative magnetic quantum number, and green arrows depict some of the possible excitations due to phonons, compare e.g. [8].

Among others, two major processes prevent an easy use of SMMs: quantum tunneling of the magnetization

\footnotetext{
* jschnack@uni-bielefeld.de
}

between nearly degenerate levels at avoided level crossings, depicted by blue arrows in Fig. 1, as well as thermal excitations across the barrier transmitted by the phonons of the molecule or the lattice of the crystal [8-18]. A very good and recent summary is provided in Ref. [8], where particulary Figure 3 explains that the transitions imposed by direct, van Vleck, Orbach, or second-order Raman processes are assumed to be of resonant nature similar to scattering processes. Only recently this paradigm has been challenged by the observation of sub-barrier tunneling which was subsequently modeled by means of anharmonic phonons [19]. The question, what is or is not required to make a good SMM [20], got a new twist.

In the present article, we do not want to contribute to the discussion of the role of the anisotropy barrier or the various transitions harmonic or anharmonic phonons can induce [16, 21-31]. Instead, we want to contribute a new question, namely whether spin-phonon interactions can open a tunneling gap and thus enable temperatureindependent groundstate tunneling of the magnetization.

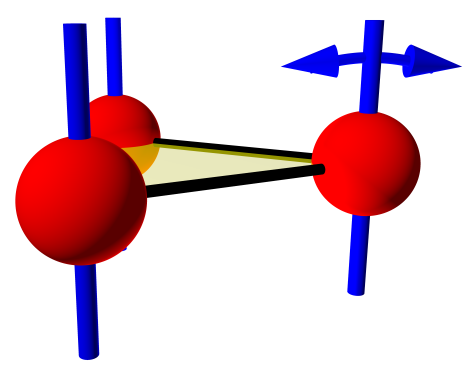

Figure 2. Model system investigated in this article: red spheres denote spins that interact ferromagnetically with their neighbors, blue sticks represent single-ion easy-axis anisotropies. The latter are allowed to vibrate.

In order to answer this question, we are going to investigate the model system shown in Fig. 2. Three spins at the corners of a triangle interact with each other ferromagnetically. In addition, each spin is subject to an easy-axis anisotropy. In our model, all three anisotropy 
axes are coupled to harmonic oscillators and can thus fluctuate away from their uniaxial rest direction. Such a simple, but yet realistic model leads directly to the opening of a tunneling gap. Consequently, a unitary time evolution of a state initialized at negative magnetic field results in magnetization tunneling at zero field due to Landau-Zener transitions [6].

The paper is organized as follows. In Section II, we introduce the model together with our numerical procedures. We present our numerical results in Section III. The article closes with a discussion in Section IV.

\section{METHOD}

The central idea of this paper is to investigate a combined quantum system of spins and phonons by numerically exactly diagonalizing an appropriate Hamiltonian. For the purpose of demonstrating the ability of phonons to open a tunneling gap, we choose a scenario where the easy anisotropy axes are coupled to vibrations of the molecule [32]. As shown schematically in Fig. 2, our system consists of three spins $s=1$ which couple ferromagnetically with each other and are subject to a singleion easy-axis anisotropy. Each of the anisotropy axes is coupled to its own harmonic oscillator and thus vibrates independently of the other axes. Thus, the total Hamiltonian of our model consists of four parts, a Heisenberg term, the single-ion anisotropy, the harmonic oscillators, and the Zeeman term:

$$
\begin{aligned}
& \underset{\sim}{H}=-2 J(\underset{\sim}{\overrightarrow{\vec{s}}} \cdot \underset{\sim}{\overrightarrow{\vec{s}}}+\underset{\sim}{\overrightarrow{\vec{s}}} \cdot \underset{\sim}{\overrightarrow{\vec{s}}}+\underset{\sim}{\overrightarrow{\vec{s}}} \cdot \underset{\sim}{\overrightarrow{\vec{s}}}) \\
& +\underset{\sim}{\vec{s}} \cdot \mathbf{D}_{1}\left({\underset{\sim}{\theta}}_{1}\right) \cdot \underset{\sim}{\vec{s}}+\underset{\sim}{\vec{s}} \cdot \mathbf{D}_{2}\left({\underset{\sim}{\theta}}_{2}\right) \cdot \underset{\sim}{\vec{s}} \\
& +\underset{\sim}{\vec{s}} \cdot \mathbf{D}_{3}\left({\underset{\sim}{\theta}}_{3}\right) \cdot \underset{\sim}{\vec{s}} \\
& +\omega_{1}\left(\underset{\sim}{a_{\sim}^{\dagger}} a_{\sim}+\frac{1}{2}\right)+\omega_{2}\left(\underset{\sim}{a_{2}^{\dagger}} a_{2}+\frac{1}{2}\right) \\
& +\omega_{3}\left(\underset{\sim}{a} \underset{\sim}{\dagger} a_{3}+\frac{1}{2}\right) \\
& +g \mu_{B} \cdot \vec{B} \cdot\left(\underset{\sim}{\overrightarrow{\vec{s}_{1}}}+\underset{\sim}{\overrightarrow{\vec{s}_{2}}}+\underset{\sim}{\overrightarrow{\vec{s}_{3}}}\right) .
\end{aligned}
$$

Here, $\underset{\sim}{\vec{s}}$ are the spin-vector operators of the three spins; $J>0$ denotes the ferromagnetic coupling. The $\mathbf{D}_{i}\left({\underset{\sim}{\theta}}_{i}\right)$ model the easy anisotropy axes

$$
\begin{aligned}
\mathbf{D}_{i}\left({\underset{\sim}{\theta}}_{i}\right) & =D \vec{e}_{i}\left({\underset{\sim}{\theta}}_{i}, \phi_{i}\right) \otimes \vec{e}_{i}\left({\underset{\sim}{\theta}}_{i}, \phi_{i}\right) \\
\vec{e}_{i}\left({\underset{\sim}{i}}_{i}, \phi_{i}\right) & =\left(\begin{array}{c}
\cos \left(\phi_{i}\right) \sin \left({\underset{\sim}{\theta}}_{i}\right) \\
\sin \left(\phi_{i}\right) \sin \left({\underset{\sim}{i}}_{i}\right) \\
\cos \left({\underset{\sim}{\theta}}_{i}\right)
\end{array}\right) \\
\phi_{i} & =(i-1) 2 \pi / 3, \quad i \in\{1,2,3\} .
\end{aligned}
$$

They depend on two angular coordinates of which $\underset{\sim}{\theta_{i}}$ couples to the harmonic oscillator degrees of freedom

$$
\begin{gathered}
\underset{\sim}{\theta_{i}=} \theta_{i, 0}+\alpha \sqrt{\omega}{\underset{\sim}{x}}_{i}, \quad i \in\{1,2,3\} \\
x_{i} \propto\left(\underset{\sim}{a}{ }_{i}^{\dagger}+{\underset{\sim}{a}}_{i}\right) .
\end{gathered}
$$

We assume the same coupling strength $\alpha$ for all three anisotropy axes with their respective oscillators and the same frequency $\omega$ for all of these oscillators. It is obvious that this model can be easily generalized to less idealized cases.

Without loss of generality, we choose a ferromagnetic exchange of $J=10 \mathrm{~K}$ and an easy anisotropy of $D=$ $-5 \mathrm{~K}$ for the following calculations. The external field will always point in global $z$-direction, i.e. $\vec{B}=B \vec{e}_{z}$.

For numerical diagonalization, we have to represent Hamiltonian (1) with respect to a basis. We use the product basis $\left\{\left|m_{1}, m_{2}, m_{3} ; n_{1}, n_{2}, n_{3}\right\rangle\right\}$, where $m_{i}=-1,0,1$ are the magnetic quantum numbers of the individual spins and $n_{i}=0,1, \ldots$ are the quantum numbers of the three oscillators. Matrix elements containing sine or cosine functions of $\underset{\sim}{\theta_{i}}$ can be evaluated exactly with the help of an intermediate basis transform. The dimension of the underlying Hilbert space is infinite due to the harmonic oscillators. We therefore cut the oscillator quantum numbers at some $n_{\max }$ for our numerical treatment. We investigated carefully that this indeed does not influence the qualitative conclusions of the present paper. Under these conditions, it turns out that $n_{\max }=1$ is sufficient to demonstrate our main finding, the opening of a tunneling gap.

The numerical solutions of the stationary as well as of the time-dependent Schrödinger equation presented in the next section have been obtained with Mathematica ${ }^{\text {TM }}$ in a recent thesis [33].

\section{NUMERICAL RESULTS}

The interesting case is given by a situation where the anisotropy axes are at rest and point uniaxially along the field direction, which is chosen in positive global $z$ direction. This means $\theta_{i, 0}=0, \forall i$. Without phonons, such a system would possess a perfect crossing of the two degenerate ground state levels at $B=0$, and thus would not show any magnetization tunneling.

If the coupling between spins and phonons is switched on, one observes two results. Each level of the spin Hamiltonian splits into a bunch of combined spin-phonon levels, and level crossings, e.g. of the ground state, turn into avoided level crossings. The width of a bunch, compare top of Fig. 3, depends both on $\alpha$ and $\omega$. The number of levels in a bunch is given by the dimension of the phonon subspace, in our calculation 8 (but the levels are degenerate). The bottom of Fig. 3 shows the behavior of the lowest levels at the avoided level crossing. The energy difference at $B=0$ is the tunnel splitting $E_{G}$

The size of the tunneling gap $E_{G}$ depends strongly on $\alpha$ and rather weakly on $\omega$, as can be seen in Fig. 4 . For values $E_{G}<10^{-13}$, the numerical determination of the tunneling gap is no longer accurate. For values of $\alpha$ in the range from 0.02 to 0.5 , the values of the tunneling gap $E_{G}$ span nine orders of magnitude. This means that vibrations of the anisotropy tensors can have a mas- 


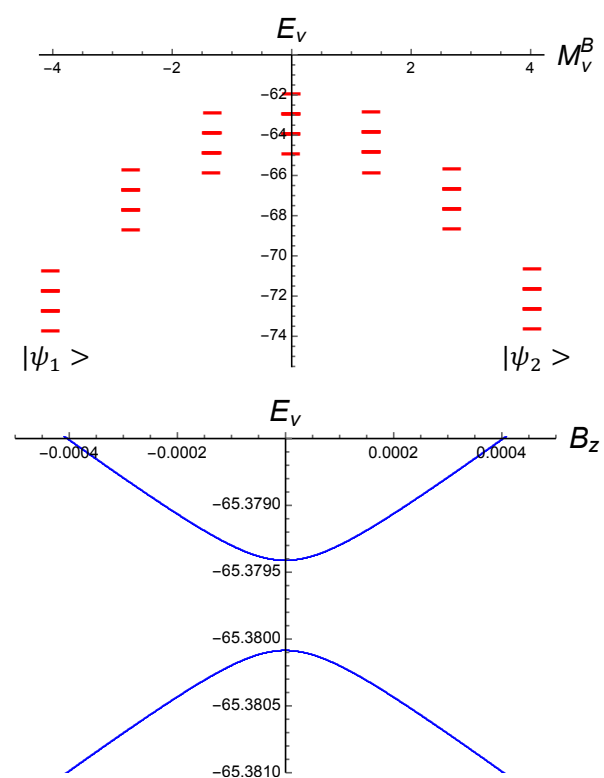

Figure 3. Top: Energy levels vs. magnetic moment $\mathcal{M}_{\nu}^{B}=$ $-g \mu_{B}\left\langle\nu\left|S^{z}\right| \nu\right\rangle$ for $\alpha=0.01, n_{\max }=1, \omega=1$. The two nearly degenerate ground states are denotes as $\left|\Psi_{1}\right\rangle$ and $\left|\Psi_{2}\right\rangle$. Bottom: Avoided level crossing of the lowest doublet for $\alpha=0.5$. The energy difference at $B=0$ is the tunnel splitting $E_{G}$.
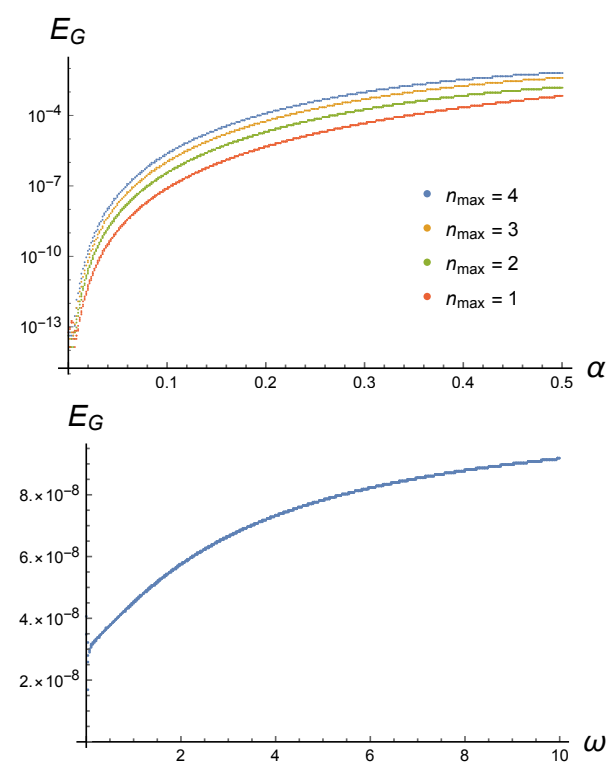

Figure 4. Size of the tunnel splitting $E_{G}$. Top: $E_{G}$ vs. $\alpha$ for $\omega=5$ and $n_{\max } \in\{1,2,3,4\}$. Bottom: $E_{G}$ vs. $\omega$ for $\alpha=0.1$ and $n_{\max }=1$.

sive influence on the character of the low-lying energy spectrum. This tendency increases with the number of available phonons, i.e. the number of oscillator excitations. A larger number of available oscillator excitations corresponds to a larger quantum mechanical (as well as thermal) variance of the deflection of all anisotropy ten- sors.

Finally, we calculate the time evolution for an initial state for various sweep rates $\dot{B}$ of the external magnetic field. For our calculations, we choose $\alpha=0.5, \omega=5$ and $n_{\max }=1$. This results in a relatively strong coupling between oscillators and anisotropy axes, but we choose this for educational reasons since the effect is stronger and therefore easier to observe. For smaller couplings $\alpha$, it will of course also be present, just less obvious.
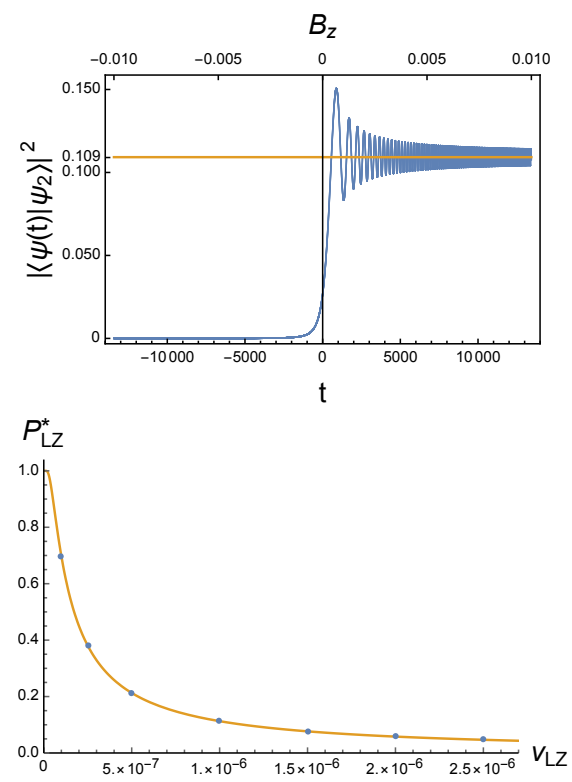

Figure 5. Landau-Zener transitions in the case of strong coupling $\alpha=0.5$ and $\omega=5$ for $n_{\max }=1$. The initial state is $\left|\Psi_{1}\right\rangle$. Top: Example of the unitary time evolution with $\dot{B}=1 \cdot 10^{-6}$. Bottom: Transition probability at the avoided crossing of the two ground state levels as a function of $\dot{B}$. Blue dots are numerical integrations, the solid curve corresponds to the Landau-Zener theory.

Figure 5 shows the time evolution of the overlap between the time-evolved state $|\Psi(t)\rangle$ and the asymptotic state $\left|\Psi_{2}\right\rangle$ the system tunnels into for a certain sweep rate $\dot{B}$ of the external field (top). The time evolution starts with $\left.\mid \Psi\left(t_{\text {start }}\right\rangle\right)=\left|\Psi_{1}\right\rangle$, see Fig. 3, at some initial time and negative external field. While passing $B=0$, the system undergoes a Landau-Zener transition and oscillates about a limiting overlap with the state $\left|\Psi_{2}\right\rangle$, compare Fig. 5. Such time evolutions have been performed for several sweep rates. The resulting transition rates, i.e. the probabilities to find the system in the other limiting state, are given by blue dots in Fig. 5 (bottom). These numerical values coincide perfectly with the results obtained from Landau-Zener theory which also tells us that the higher-lying excited states of the system do not really mix in at the transition. With that confidence, one can now read off the transition probabilities for smaller sweep rates that are not accessible in numerical time evolutions due to the prohibitively large number of time steps one would have to perform. 

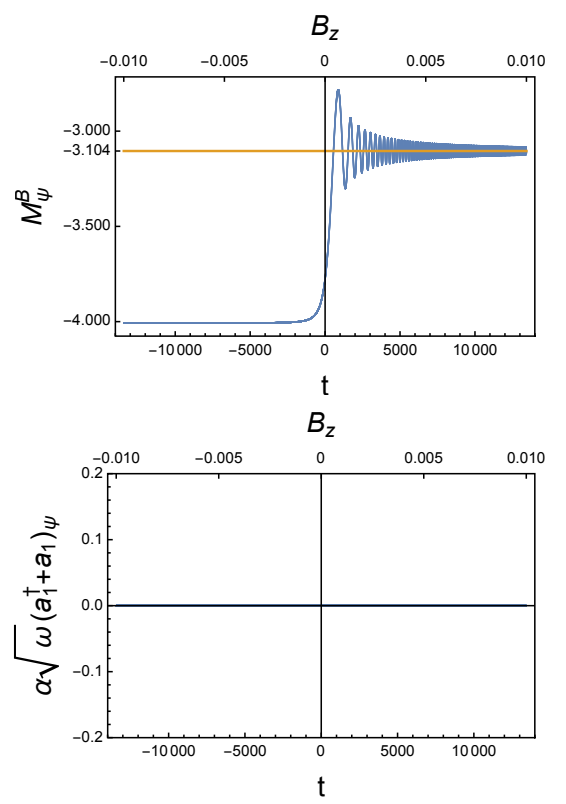

Figure 6. Landau-Zener transitions in the case of strong coupling $\alpha=0.5$ and $\omega=5$ for $n_{\max }=1$. The initial state is $\left|\Psi_{1}\right\rangle$. Top: Magnetization tunneling during the unitary time evolution with $\dot{B}=1 \cdot 10^{-6}$. Bottom: Expectation values of $\alpha \sqrt{\omega}\left(\underset{\sim}{a}+\underset{\sim}{a_{1}}\right) \propto \underset{\sim}{\theta_{1}} .{\underset{\sim}{\theta}}_{2}$ and $\underset{\sim}{\theta_{3}}$ behave in the same way. All numerical values are virtually zero.

Finally, we want to discuss the behavior of the phonon subsystem, i.e. the dynamics of the anisotropy axes. For the time evolution depicted in Fig. 5, where one of the two nearly degenerate ground states is taken as initial state and evolved via a time-dependent magnetic field, we can not only evaluate the amount of magnetization that tunnels. We can also calculate the phononic excitations, i.e. the vibrations of the anisotropy axes while the system is swept across $B=0$. Both $\left|\Psi_{1}\right\rangle$ and $\left|\Psi_{2}\right\rangle$ are practically in their phononic ground states, i.e. the phononic contribution to these eigenstates of the total Hamiltonian is dominantly $\left|n_{1}=0, n_{2}=0, n_{3}=0\right\rangle$. It turns out that this does not really change in the course of the time evolution, i.e. phonons are not excited and the anisotropy axes do not vibrate noticibly, nevertheless some part of the magnetization tunnels, compare Figure 6 .

We also considered a superposition of initial states that belong to a bunch of levels which may be simultaneously occupied at non-zero temperature. For this purpose we chose a smaller $\omega=0.1$. The situation is displayed in Fig. 7. Although the anisotropy axes vibrate fiercely about the $z$-direction with a high internal frequency and a much larger amplitude, the effect on the magnetization tunneling is only slightly bigger compared to a situation with only $\left|\Psi_{1}\right\rangle$ as initial state but adapted smaller $\omega=0.1$ (not shown). We suppose that the internal frequency of the phonon subsystem is just too big in the present scenario, so that the spin system is too inert to
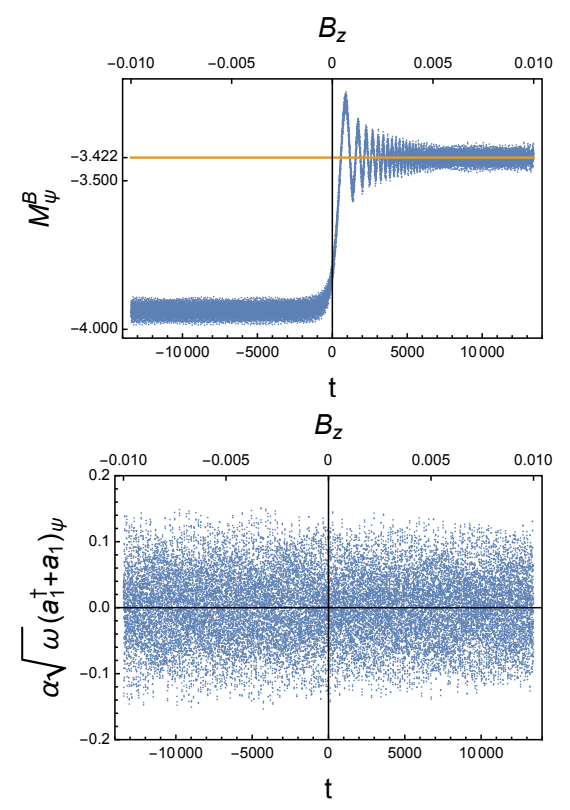

Figure 7. Landau-Zener transitions in the case of strong coupling $\alpha=0.5$ and $\omega=0.1$ for $n_{\max }=1$. The initial state is a supersosition of all state in the bunch of $\left|\Psi_{1}\right\rangle$, see Fig. 3. Top: Magnetization tunneling during the unitary time evolution with $\dot{B}=1 \cdot 10^{-6}$. Bottom: Expectation values of $\alpha \sqrt{\omega}\left(\underset{\sim}{a_{1}^{\dagger}}+{\underset{\sim}{a}}_{1}\right) \propto \underset{\sim}{\theta_{1}} .{\underset{\sim}{\theta}}_{2}$ and ${\underset{\sim}{\theta}}_{3}$ behave in the same way. The dynamics contains very high frequencies due to which the plots look fuzzy. Each plot shows 20,000 points which corresponds to $\Delta t \approx 1$ between points. Mathematica ${ }^{\mathrm{TM}}$ uses a variable step size that is much smaller.

\section{follow.}

The last investigations lead us to the final and important conclusion that the mere coupling $\alpha$ of the spins to the zero-point motion of the oscillator/phonon subsystem is sufficient to open the tunneling gap and induce Landau-Zener transitions. Although the expectation value of the anisotropy axes does not deviate from the uniaxial $z$-direction, the fact that this value is not sharp but subject to a quantum mechanical variance leads to the reduction of bistability.

\section{DISCUSSION AND CONCLUSIONS}

In this article, we were able to demonstrate that phonons can open up a tunneling gap between otherwise degenerate ground states of a single-molecule magnet. In our model calculation, the phonons destroyed the uniaxial character of the single-ion easy-axis anisotropies. We expect that phonons breaking the rotational symmetry about the common axis of the field and the anisotropy tensors in any other way would result in a similar effect.

Our investigations are in line with earlier studies of spin-phonon interactions, for instance in Ref. [34], where the phonons that modify the exchange integrals are treated classically as well as in Ref. [35] where a 
distortion-dependent Dzyaloshinskii-Moriya interaction is considered. Despite having a different focus, both papers agree with our observation that if the spin-phonon coupling is too strong, static distortions of the system occur since the combined system of spins and lattice becomes spin-Peierls unstable.

Summarizing, we would like to state that investigations such as the present one help determining prerequisites for the successful design of single-molecule magnets by identifying harmful vibrations that should be suppressed in such molecules.

\section{ACKNOWLEDGMENT}

This work was supported by the Deutsche Forschungsgemeinschaft DFG (314331397 (SCHN 615/23-1); 355031190 (FOR 2692); 397300368 (SCHN 615/25-1)). We thank Patrick Vorndamme for carefully reading the manuscript.
[1] R. Sessoli, D. Gatteschi, A. Caneschi, and M. A. Novak, "Magnetic bistability in a metal-ion cluster," Nature 365, 141 (1993).

[2] J. R. Friedman, M. P. Sarachik, J. Tejada, and R. Ziolo, "Macroscopic Measurement of Resonant Magnetization Tunneling in High-Spin Molecules," Phys. Rev. Lett. 76, 3830 (1996).

[3] L. Thomas, F. Lionti, R. Ballou, D. Gatteschi, R. Sessoli, and B. Barbara, "Macroscopic quantum tunnelling of magnetization in a single crystal of nanomagnets," Nature 383, 145 (1996).

[4] F. Lionti, L. Thomas, R. Ballou, B. Barbara, A. Sulpice, R. Sessoli, and D. Gatteschi, "Thermally assisted macroscopic quantum resonance on a single crystal of Mn-12acetate," J. Appl. Phys. 81, 4608 (1997).

[5] I. Chiorescu, W. Wernsdorfer, A. Müller, H. Bögge, and B. Barbara, "Butterfly hysteresis loop and dissipative spin reversal in the $S=1 / 2, \mathrm{~V}_{15}$ molecular complex," Phys. Rev. Lett. 84, 3454 (2000).

[6] W. Wernsdorfer, R. Sessoli, A. Caneschi, D. Gatteschi, A. Cornia, and D. Mailly, "Landau-Zener method to study quantum phase interference of $\mathrm{Fe}_{8}$ molecular nanomagnets," J. Appl. Phys. 87, 5481 (2000).

[7] D. Gatteschi and R. Sessoli, "Quantum tunneling of magnetization and related phenomena in molecular materials," Angew. Chem., Int. Edit. 42, 268 (2003).

[8] S. T. Liddle and J. van Slageren, "Improving f-element single molecule magnets," Chem. Soc. Rev. 44, 6655 (2015).

[9] M. N. Leuenberger and D. Loss, "Spin tunneling and phonon-assisted relaxation in Mn12-acetate," Phys. Rev. B 61, 1286 (2000).

[10] I. Chiorescu, R. Giraud, A. G. M. Jansen, A. Caneschi, and B. Barbara, "Phonon-Assisted Tunneling in the Quantum Regime of Mn12 Acetate," Phys. Rev. Lett. 85, 4807 (2000).

[11] E. M. Chudnovsky, D. A. Garanin, and R. Schilling, "Universal mechanism of spin relaxation in solids," Phys. Rev. B 72, 094426 (2005).

[12] M. Takahata, M. Shoji, H. Nitta, R. Takeda, S. Yamanaka, M. Okumura, M. Nakano, and K. Yamaguchi, "Quantum dynamics in high-spin molecules, spin dendrimers, and spin lattices," Int. J. Quant. Chem. 105, 615 (2005).

[13] M. Takahata, M. Shoji, S. Yamanaka, M. Nakano, and K. Yamaguchi, "Formulation of master equation approach involving spin-phonon coupling: Toward an understanding of spin dynamics in magnetic dendrimers,"
Polyhedron 24, 2653 (2005).

[14] S. J. Blundell, "Molecular magnets," Contemp. Phys. 48, 275 (2007).

[15] A. Bianchi, S. Carretta, P. Santini, G. Amoretti, J. Lago, M. Corti, A. Lascialfari, P. Arosio, G. Timco, and R. E. P. Winpenny, "Phonon-induced relaxation in the $\mathrm{Cr}_{7} \mathrm{Ni}$ magnetic molecule probed by NMR," Phys. Rev. B 82, 134403 (2010).

[16] T. Glaser, "Rational design of single-molecule magnets: a supramolecular approach," Chem. Commun. 47, 116 (2011).

[17] J.-P. Venne, B. Feldscher, S. Walleck, A. Stammler, H. Bögge, J. Schnack, and T. Glaser, "Rational Improvement of Single-Molecule Magnets by Enforcing Ferromagnetic Interactions," Chem. Eur. J. 25, 4992 (2019).

[18] J. Schnack, "Large magnetic molecules and what we learn from them," Contemp. Phys. 60, 127 (2019).

[19] A. Lunghi, F. Totti, R. Sessoli, and S. Sanvito, "The role of anharmonic phonons in under-barrier spin relaxation of single molecule magnets," Nat. Commun. 8, 14620 (2017).

[20] F. Neese and D. A. Pantazis, "What is not required to make a single molecule magnet," Faraday Discuss. 148, 229 (2011).

[21] O. Waldmann, "A Criterion for the Anisotropy Barrier in Single-Molecule Magnets," Inorg. Chem. 46, 10035 (2007).

[22] E. Ruiz, J. Cirera, J. Cano, S. Alvarez, C. Loose, and J. Kortus, "Can large magnetic anisotropy and high spin really coexist?" Chem. Commun. , 52 (2008).

[23] D. E. Freedman, W. H. Harman, T. D. Harris, G. J. Long, C. J. Chang, and J. R. Long, "Slow Magnetic Relaxation in a High-Spin Iron(II) Complex," J. Am. Chem. Soc. 132, 1224 (2010).

[24] B. Bechlars, D. M. D'Alessandro, D. M. Jenkins, A. T. Iavarone, S. D. Glover, C. P. Kubiak, and J. R. Long, "High-spin ground states via electron delocalization in mixed-valence imidazolate-bridged divanadium complexes," Nat. Chem. 2, 362 (2010).

[25] K. S. Pedersen, J. Dreiser, H. Weihe, R. Sibille, H. V. Johannesen, M. A. Sørensen, B. E. Nielsen, M. Sigrist, H. Mutka, S. Rols, J. Bendix, and S. Piligkos, "Design of Single-Molecule Magnets: Insufficiency of the Anisotropy Barrier as the Sole Criterion," Inorg. Chem. 54, 7600 (2015).

[26] C. A. P. Goodwin, F. Ortu, D. Reta, N. F. Chilton, and D. P. Mills, "Molecular magnetic hysteresis at 60 kelvin in dysprosocenium," Nature 548, 439 (2017). 
[27] L. Escalera-Moreno, J. J. Baldovi, A. Gaita-Arino, and E. Coronado, "Spin states, vibrations and spin relaxation in molecular nanomagnets and spin qubits: a critical perspective," Chem. Sci. 9, 3265 (2018).

[28] D. Reta and N. F. Chilton, "Uncertainty estimates for magnetic relaxation times and magnetic relaxation parameters," Phys. Chem. Chem. Phys. 21, 23567 (2019).

[29] F. Neese, M. Atanasov, G. Bistoni, D. Maganas, and S. Ye, "Chemistry and Quantum Mechanics in 2019: Give Us Insight and Numbers," J. Am. Chem. Soc. 141, 2814 (2019).

[30] A. Lunghi and S. Sanvito, "How do phonons relax molecular spins?" Science Advances 5, eaax7163 (2019).

[31] A. Albino, S. Benci, L. Tesi, M. Atzori, R. Torre, S. Sanvito, R. Sessoli, and A. Lunghi, "First-Principles Investigation of SpinPhonon Coupling in Vanadium-Based
Molecular Spin Quantum Bits," Inorg. Chem. 58, 10260 (2019).

[32] A. Lunghi, F. Totti, S. Sanvito, and R. Sessoli, "Intramolecular origin of the spin-phonon coupling in slowrelaxing molecular magnets," Chem. Sci. 8, 6051 (2017).

[33] K. Irländer, Spin-Phonon-Wechselwirkung am Beispiel eines Spin-1-Trimers mit Einzelionen-Anisotropie, Master's thesis, Bielefeld University (2020).

[34] M. Elhajal and F. Mila, "Dynamic spin Jahn-Teller effect in small magnetic clusters," Eur. Phys. J. B 47, 185 (2005).

[35] V. Lante, I. Rousochatzakis, K. Penc, O. Waldmann, and F. Mila, "Spin-Peierls instabilities of antiferromagnetic rings in a magnetic field," Phys. Rev. B 79, 180412 (2009). 